\title{
VL30 retrotransposition signals activation of a caspase- independent and p53-dependent death pathway associated with mitochondrial and lysosomal damage
}

Dimitrios Noutsopoulos ${ }^{1}$, Georgios Markopoulos ${ }^{1}$, Georgios Vartholomatos ${ }^{2}$, Evangelos Kolettas ${ }^{3}$, Nicolaos Kolaitis ${ }^{2}$, Theodore Tzavaras ${ }^{1}$

${ }^{I}$ Laboratory of General Biology, University of Ioannina, Medical School, 45110 Ioannina, Greece; ${ }^{2}$ Unit of Molecular Biology, Hematology Laboratory, University Hospital of Ioannina, 45110 Ioannina, Greece, ${ }^{3}$ Cell and Molecular Physiology Unit, Laboratory of Experimental Physiology, University of Ioannina, Medical School, 45110 Ioannina, Greece

The impact of long terminal repeat (LTR) retrotransposition on cell fate is unknown. Here, we investigated the effect of VL30 retrotransposition on cell death in SV40-transformed mouse SVTT1 cells. Transfection of a VL30 retrotransposon decreased the clonogenicity of SVTT1 by 17-fold, as compared to parental NIH3T3 cells. Correlated levels of retrotransposition frequency and cell death rates were found in retrotransposition-positive SVTT1 cloned cells, exhibiting DNA fragmentation, nuclear condensation, multinucleation and cytoplasmic vacuolization. Analysis of activation of effector caspases revealed a caspase-independent cell death mechanism. However, cell death was associated with p53 induction and concomitant upregulation of PUMA $\alpha$ and Bax and downregulation of Bcl-2 and Hsp70 protein expression. Moreover, we found partial loss of colocalization of large T-antigen (LT)/p53 and p53 translocation to mitochondria, leading to mitochondrial outer membrane permeabilization (MOMP) accompanied by lysosomal membrane permeabilization (LMP). Interestingly, treatment with the antioxidant $N$-acetylcysteine abolished cell death, suggesting the involvement of mitochondrial-derived reactive oxygen species, and resulted in an increase of retrotransposition frequency. Importantly, the induction of cell death was VL30 retrotransposon-specific as VL30 mobilization was induced; in contrast, mobilization of the non-LTR L1 (LINE-1, long interspersed nuclear element-1), B2 and LTR MusD retrotransposons decreased. Our results provide, for the first time, strong evidence that VL30 retrotransposition mediates cell death via mitochondrial and lysosomal damage, uncovering the role of retrotransposition as a nuclear signal activating a mitochondrial-lysosomal crosstalk in triggering cell death.

Keywords: retrotransposon, VL30, retrotransposition, cell death, p53, mitochondrion, lysosome

Cell Research (2010) 20:553-562. doi:10.1038/cr.2010.48; published online 13 April 2010

\section{Introduction}

Retrotransposons are singular repetitive elements inte-

Correspondence: Theodore Tzavaras

Tel: +0030-26510-07569; Fax: +0030-26510-07863

E-mail: thtzavar@cc.uoi.gr

Abbreviations: VL30s (viral-like 30 elements); NAC ( $N$-acetylcysteine); ROS (reactive oxygen species); EGFP (enhanced green fluorescent protein); FACS (fluorescence-activated cell scanning); LTR (long terminal repeat); LINE-1 (L1) (long interspersed nuclear element-1); LT (large Tantigen); MOMP (mitochondrial outer membrane permeabilization); LMP (lysosomal membrane permeabilization)

Received 20 July 2009; revised 8 December 2009; accepted 4 January 2010, published online 13 April 2010 grated in the mammalian genome. They comprise $37.3 \%$ of the mouse genome and are represented by the subclasses of non-long terminal repeat (LTR) and LTR retrotransposons [1]. Mouse VL30s are an LTR retrotransposon family of $\sim 200$ members resembling retroviruses [2] and their RNA expression is induced by a variety of agents [2, 3], among them SV40 virus [4]. Isolated VL30 sequences are characterized by multiple stop codons [5], and thus in the absence of coding capacity, they are classified as non-autonomous LTR retrotransposons [2].

Several lines of evidence document the importance of VL30s. Their expression enhances the metastatic potential of human melanoma cells [6] through complex formation of VL30 RNA with PSF. PSF is a repressor protein binding RNA in spliceosomes, and VL30 RNA 
binding to PSF reversed PSF-mediated gene repression [7], thus affecting various processes such as embryogenesis, oncogenesis and steroidogenesis [8, 9]. Importantly, recent findings show that noncoding VL30 RNA regulates proto-oncogene transcription, cell proliferation and tumorigenesis in mice through interacting with the PSF protein [10]. Remarkably, in experimentally induced cerebral ischemia in mouse, VL30 transcripts were induced and found to bind polyribosomes, suggesting an effect of VL30s on polyribosome stability [11].

Retrotransposition is an intracellular process requiring a retrotransposon RNA-intermediate [12], which is reverse transcribed into cDNA followed by integration into a new genomic site. Importantly, such new integrations can affect diverse genome functions and genome integrity at various levels. Retrotransposition events can cause human and mouse diseases [13] and affect telomere stability, chromosomal distribution during cell division, chromatin organization and timing of gene expression in development [14].

Programmed cell death (PCD) is an important developmental and homeostatic process leading to elimination of damaged cells through different, biochemically and morphologically distinct death types. Apoptosis represents such a type of PCD that is elicited through two main pathways: the extrinsic and intrinsic ones, also known as the 'death receptor' (external) and 'mitochondrial' (internal) pathway, respectively $[15,16]$. Although apoptosis is usually dependent on caspase activation [15] cell death can also occur in a caspase-independent manner [17], while the p53 protein usually plays a pivotal role in both cases [15]. Increasing evidence suggests that different cell death types may overlap with particular characteristics displayed simultaneously [17].

So far, evidence for the impact of retrotransposition on PCD mainly comes from human non-LTR retrotransposons L1 (LINE-1, long interspersed nuclear element-1). An early observation reported suicidal death characterized by sudden transcriptional activation and concomitant induced retrotranspositions [18]. It was also shown that UV light- and ionizing radiation-induced L1 retrotransposition caused PCD in rat chloro-leukemia cells [19]. Recently, it was reported that L1 retrotransposition induced apoptosis in human cancer cells [20, 21]. Regarding mouse LTR retrotransposons, we reported that high induction of VL30 retrotransposition resulted in massive cell death or cytotoxic effects in NIH3T3 cells following either expression of SV40 large T-antigen (LT) [22] or vanadium-generated oxidative stress [23], respectively. Although these data demonstrated the effect of retrotransposition on $\mathrm{PCD}$, the underlying mechanism remains to be elucidated.
In this report, we investigated the effects of LTR retrotransposition on cell fate of SV40-transformed NIH3T3 cells. We found that VL30 retrotransposition specifically mediated cell death in a caspase-independent and p53dependent manner by activating the mitochondrial death pathway in association with lysosomal membrane permeabilization (LMP).

\section{Results}

\section{VL30 retrotransposition is correlated with cell death}

Our previous studies, demonstrating massive cell death associated with the induction of VL30 retrotransposition in SV40-transformed NIH3T3 cells [22], prompted us to investigate in detail the effect of LTR retrotransposition on cell death.

We first determined the clonogenic efficiency of SV40transformed SVTT1 versus normal control NIH3T3 cells following transfection of an EGFP-tagged VL30 element NVL-3*/EGFP-INT [22] or plasmid EGFP-N1 (as a control for EGFP expression, since retrotranspositions are measured through EGFP-positive cells) [22]. Transfected NIH3T3 cells produced a large number of clones, although with non-statistically significant difference $(P 1=$ 0.295 ), showing the efficiency of both plasmids in producing high numbers of foci. Interestingly, while a large number of foci was obtained with pEGFP-N1 in SVTT1 cells, we found a $\sim 17$-fold decrease in the number of colonies obtained using pNVL-3*/EGFP-INT $(P 2<0.001)$ (Figure 1A). Given that VL30 retrotransposition occurs at a high frequency in SVTT1 but not in NIH3T3 cells [22], these data suggested that the resulting retrotransposition events decreased SVTT1 clonogenicity either through growth retardation or induction of cell death.

To assess whether the extent of VL30 retrotransposition events was directly related to cell death, we measured the retrotransposition frequency and cell cycle phase distribution of 10 randomly isolated pNVL-3*/ EGFP-INT-derived SVTT1 clones. We found induction of retrotransposition in all the cell clone populations, as expected [22], with frequencies ranging from $0.20 \%$ to $22.6 \%$ and values for sub-G1 populations from $5.4 \%$ to $39 \%$. Interestingly, the extent of retrotransposition events and cell death rates were related, as documented in three representative clones (Figure 1B and 1C). Specifically, clone 5 with a high retrotransposition frequency of $22.6 \%$ exhibited a concomitant high sub-G1 fraction of $\sim 39 \%$, clone 6 with a low frequency of $0.72 \%$ exhibited a low sub-G1 value of $5.46 \%$, while clone 7 showed both values at intermediate levels between those of clones 5 and 6. Collectively, these data demonstrate the correlation of induced retrotransposition events with cell death. 
A

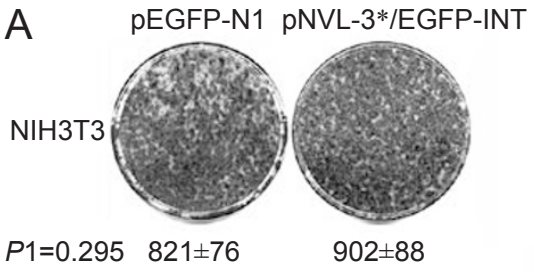

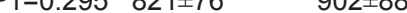

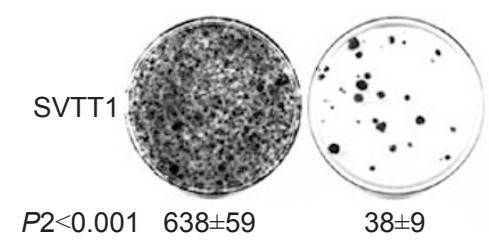

B

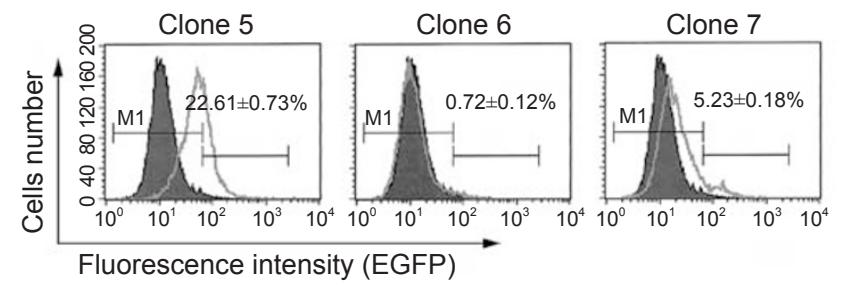

C

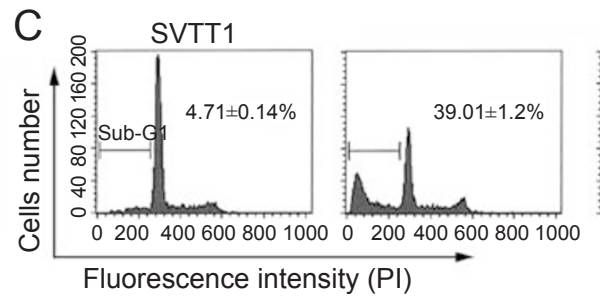

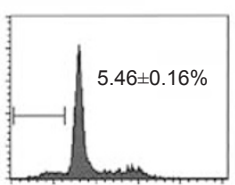

0200400600800100002004006008001000

Figure 1 Correlation of VL30 retrotransposition to cell death. (A) NIH3T3 or SVTT1 cells were transfected with pEGFP-N1 and pNVL-3*/EGFP-INT DNA, and the produced foci were stained and counted. Numbers represent the mean values of foci with indicated standard errors $(n=4)$, while $P 1$ and $P 2$ are statistical significance between foci obtained by pEGFP-N1 and pNVL-3*/EGFP-INT in NIH3T3 or SVTT1 cells, respectively. (B and C) Representative pNVL-3*/EGFP-INT-derived clone cells were measured for EGFP-positive and sub-G1 cell populations with FACS. Overlays of histogram graphs in B represent fluorescence profiles of SVTT1 (solid black) and clone cells (grey lines), respectively. Histogram graphs in C represent cell cycle phase distribution. Thresholds are shown with bars and M1 is control auto-fluorescence $(99.7 \%)$. Mean values $(n=3)$ with SD $( \pm)$ shown in $\mathbf{B}$ and $\mathbf{C}$ are net retrotransposition frequencies or cell death rates, respectively.

\section{VL30 retrotransposition-positive cells display features of cell death}

Next, we examined whether retrotransposition-positive cells displayed typical features of cell death. First, PCR analysis was performed to corroborate the retrotransposition positivity of clones 5, 6 and 7 through detecting new copies integrated in the genome. As expected, a product of $342 \mathrm{bp}$, diagnostic for retrotransposition events [22], was detected in all three clones (Figure 2A) but at different levels, which correlated with their corresponding retrotransposition frequencies (Figure 1B). In parallel, lowmolecular weight DNA from these clones and parental control SVTT1 cells were examined for DNA fragmentation. Low molecular weight-sized DNA fragments (Figure 2B) were detected with a profile showing an association of DNA fragmentation levels with the extent of VL30 retrotransposition events measured earlier (Figure 1B).

Assuming that the cell morphological changes would be best detected using cells with high retrotransposition frequency, we examined by UV microscopy clone 5 and control SVTT1 cells following staining with DAPI. In parental SVTT1 cells, an even staining was observed exclusively in the nucleus (Figure 2C, c). In contrast, clone 5 cells displayed a characteristic cytoplasmic green fluorescence (Figure 2C, e) highlighting the occurrence of retrotransposition events [22] and exhibited DAPI accumulation indicative of nuclear condensation, a hallmark of apoptosis. In addition, nuclear debris were observed throughout the culture, which could be cleared off following treatment with DNase I (data not shown), providing further evidence of nuclear fragmentation (Figure $2 \mathrm{C}$, f and i). Importantly, we found that $\sim 10 \%$ of the cell population was multinucleated (Figure $2 \mathrm{C}$, e and $\mathrm{g}$ ), suggesting mitotic catastrophe [17], and $\sim 70 \%$ of the cells were characterized by large numbers of vacuoles (Figure 2C, d and h), reminiscent of necrotic-like death. Taken together, these data show that induced VL30 retrotransposition is accompanied with features of different cell death types.

\section{VL30 retrotransposition-induced cell death is caspase- independent}

As caspases are key molecules involved in cell death, we investigated whether their activation was related to VL30 retrotransposition-induced cell death using three approaches.

First, whole protein lysates of parental untreated and staurosporine-treated SVTT1 cells were analyzed along with those extracted from the cells of clones 5,6 and 7 by immunoblotting for the expression of activated caspase-3 - a main apoptosis effector caspase [15]. In contrast to the positive control, no activated caspase- 3 was detected in either parental cells or cells of clones 5-7 (Figure 3A). Second, protein lysates of clones and parental SVTT1 cells were assayed with the fluorogenic Ac-DEVD-AMC substrate, which is proteolytically cleaved mainly by cas- 
A

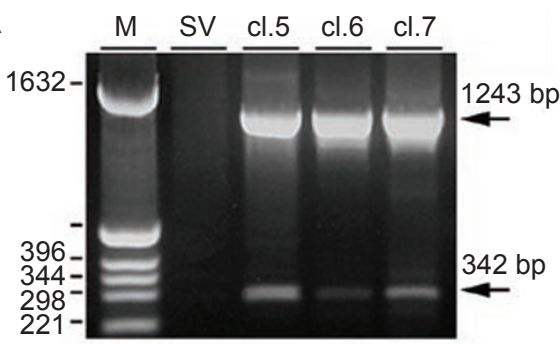

B

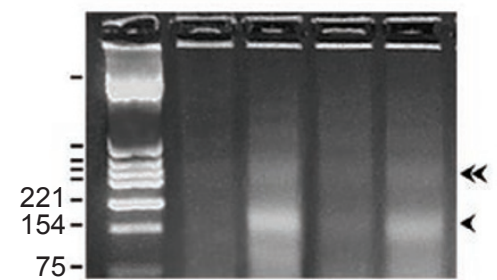

C
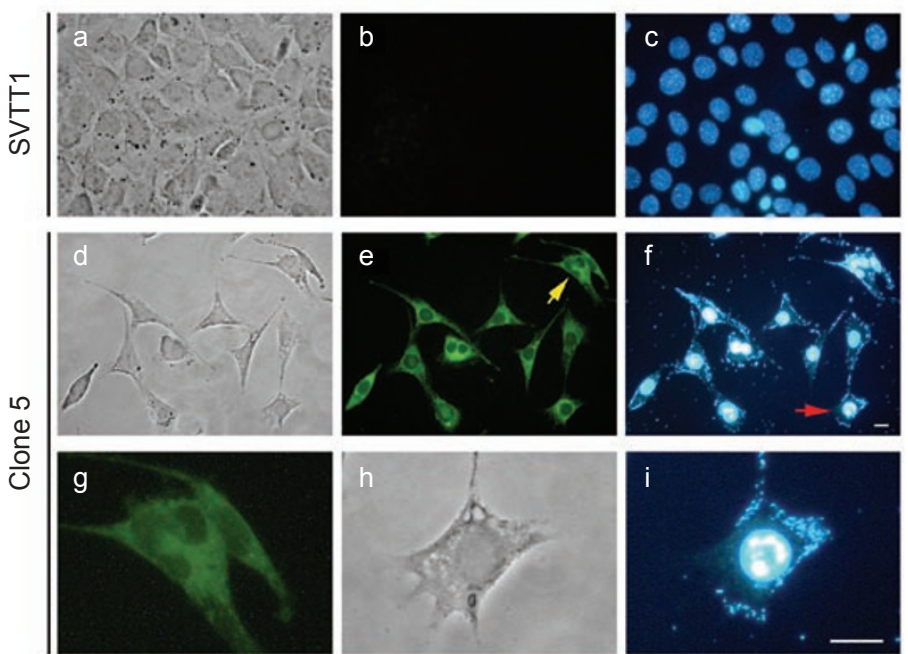

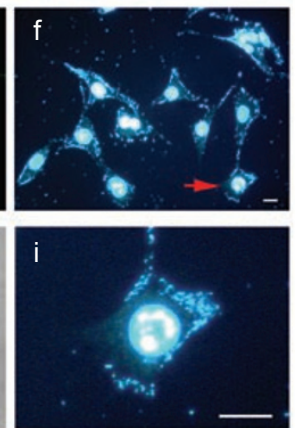

Figure 2 Cell death features of VL30 retrotransposition-positive cells. (A) PCR of DNA lysates of SVTT1 (SV) and clone cells (cl.5-7). Arrowed 1243- and 342-bp products represent pNVL-3*/EGFP-INT and retrotranspositions, respectively. M represents pBR322/Hinfl markers. (B) DNA fragmentation of samples shown in A. Single or double arrowhead shows mono- or dinucleosomes, respectively. (C) SVTT1 and clone 5 cells were fixed, stained with DAPI and photographed under normal (a, d and $h$ ) and fluorescent light with B-2A (b, e and g) or UV-2B (c, $f$ and i) filter, respectively. Panels $g, h$ and $i$ are enlargements of yellow and red arrow-indicated cells in e and $f$, respectively. Scale bars in $f$ and $i$ indicate $10 \mu \mathrm{m}$, representative of panels a-f and $g-i$, respectively.

\section{A}

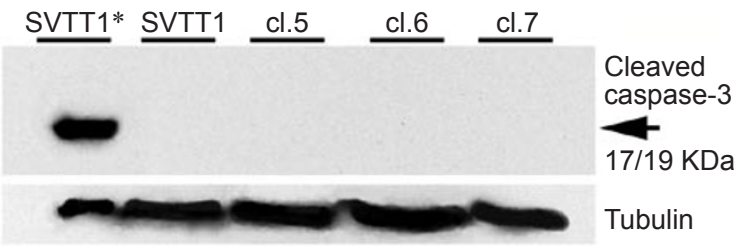

B
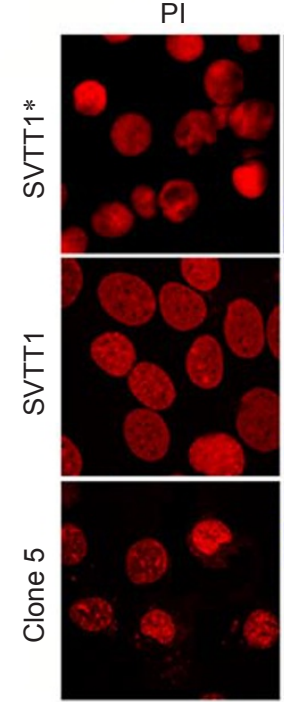

Lamin B
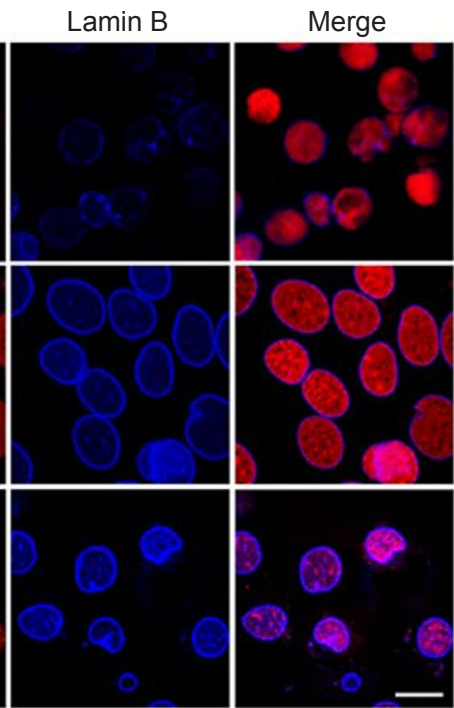

C

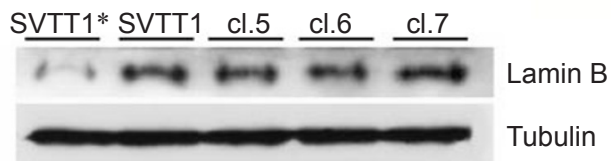

pase-3 and recognized by effector caspase-7 [24]. Biochemical analysis showed no fluorescence shift (Supplementary information, Figure S1), confirming the absence of caspase- 3 activation. Third, as lamins represent proteolysis substrates for activated caspase- 3 and caspase- 6 $[25,26]$, we performed immunofluorescence analysis for lamin B in clone 5 cells stained with propidium iodide (PI) to assess whether lamin B cleavage was associated with an altered nuclear morphology (Figure 2C, f and i). In control SVTT1 cells, both nuclei as well as the lamin B rim were normal, while staurosporine-treated SVTT1 cells exhibited both pyknotic nuclei and cleavage of lamin B. In clone 5 cells, despite nuclear condensation evidenced by PI accumulation, lamin B rim remained intact, although its dimensions were decreased (Figure 3B), suggesting the absence of proteolysis, which was further supported by immunoblotting (Figure 3C). Collectively, these data provide strong evidence that induced VL30 retrotransposition elicits a type of caspase-independent

Figure 3 Lack of caspase activation in VL30 retrotranspositioninduced cell death. (A) Immunoblotting of whole protein lysates $(100 \mu \mathrm{g})$ with an activated caspase-3 antibody. (B) Immunofluorescence of cells stained for Lamin B and with PI was visualized by confocal microscopy. Scale bar indicates $10 \mu \mathrm{m}$. (C) Immunoblotting of whole protein lysates $(50 \mu \mathrm{g})$ with a Lamin B antibody. SVTT1* represents SVTT1 cells treated with $1 \mu \mathrm{M}$ staurosporine. 
cell death.

\section{VL30 retrotransposition-induced cell death is p53-depen-} dent

Next, we investigated the expression of p53 protein, a major mediator of apoptosis in response to the stress of mammalian cells [27], in clones 5, 6 and 7 and in NIH3T3 and SVTT1 cells as controls. While higher levels of p53 - due to its stabilization by LT - were detected in SVTT1 compared to NIH3T3 cells [4, 28], p53 was induced in all three retrotransposition-positive clones compared to parental SVTT1 cells (Figure 4A). Significantly, p53 expression was $\sim$ sixfold higher in clone 5 , while that in clones 6 and 7 was at lower and intermediate levels of 1.4- and 2.5-fold, respectively (Figure 4A), in a manner that correlated with the retrotransposition extent (Figure 1B). To assess the transactivating or regulatory properties of induced p53, the expression of p53 target genes PUMA $\alpha, B c 1-2$ and Bax [29] or p53-regulated protein Hsp70 [30] was examined by immunoblotting. We found that the expression of proapoptotic PUMA $\alpha$ and Bax was induced, with the latter also being detected in its oligomerization state, while that of antiapoptotic Bcl-2 and Hsp70 was reduced (Figure 4B), in a manner that correlated with both p53 expression levels (Figure 4A) and cell death related to the retrotransposition extent (Figure $1 \mathrm{~B}$ and $1 \mathrm{C})$.
Because p53 is complexed with LT - hence its function blocked - in SVTT1 cells its induction and transactivating properties found prompted us to examine the state of the LT/p53 complex. SVTT1 and clone 5 cells stained for LT and p53 were analyzed by confocal laser scanning microscopy. In SVTT1 cells, LT and p53 proteins were strictly colocalized in the nucleus, as expected. In contrast, clone 5 cells exhibited a p53 signal not entirely colocalized with LT but instead was detected both in the nucleus and cytoplasm (Figure 4C), reminiscent of LT/p53 complex disruption and p53 redistribution similar to that observed in SV40-transformed cells exposed to oxidative stress [28]. Partial colocalization loss was further documented by Pearson's correlation and scatter plot analysis (Supplementary information, Figure S2), providing evidence that a fraction of p53 was free and functional. In summary, these data suggest that retrotransposition-induced cell death is p53-dependent.

\section{$V L 30$ retrotransposition activates the mitochondrial} death pathway and it is associated with lysosomal damage

It is reported that p53 translocates to mitochondria under stress conditions [31]. To investigate such a possibility in our cell assay system, we examined the localization of AIF and p53 in parental SVTT1 and clone 5 cells. SVTT1 cells exhibited a distinct mitochondrial and nuclear localization of AIF and p53, respectively. Impor-
A

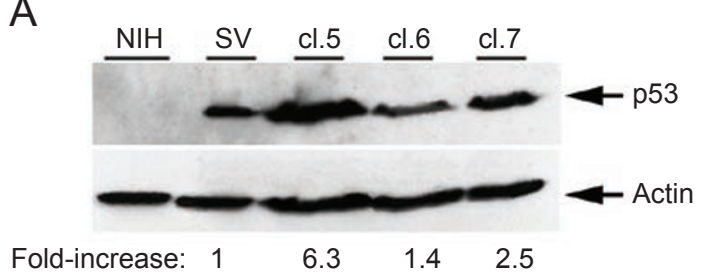

B

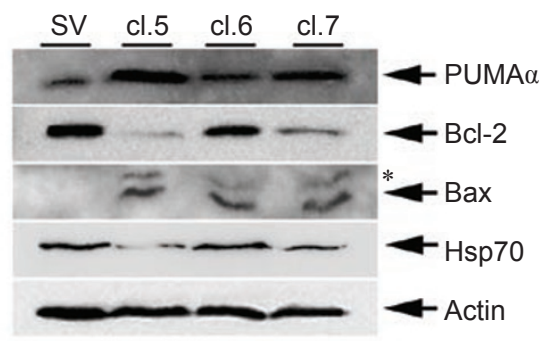

C

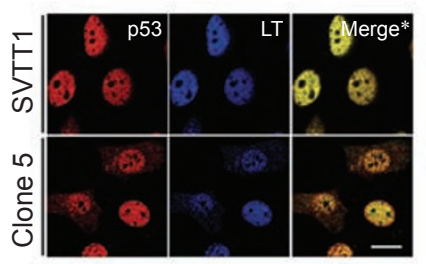

D

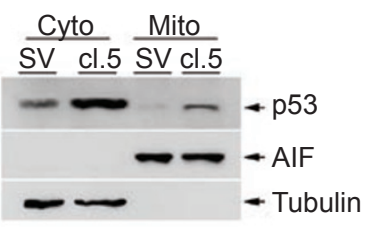

E

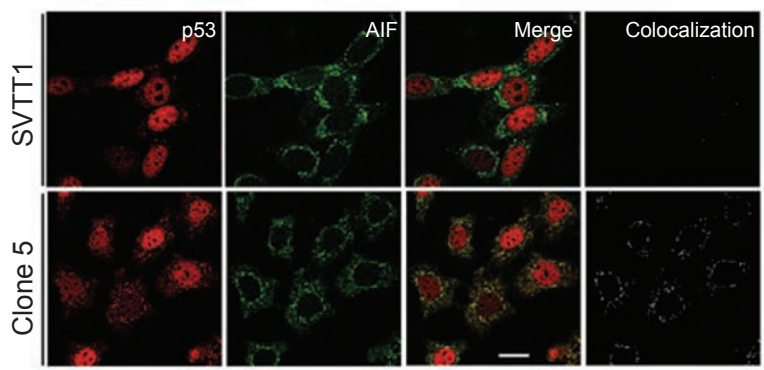

Figure 4 p53 induction, transactivation and mitochondrial translocation in VL30 retrotransposition-induced cell death. (A and B) Immunoblotting of p53, or PUMA $\alpha, \mathrm{Bcl}-2$, Bax and Hsp70 with 20 or $40 \mu \mathrm{g}$ of whole protein lysate, respectively. Asterisk in Bax panel shows oligomerization. (C) Immunofluorescence of cells stained with p53 and LT antibodies. In merge*, an asterisk denotes conversion of LT blue signal to green pseudocolor before overlay. (D) Immunoblotting of cytoplasmic (Cyto) or mitochondrial (Mito) protein fractions $(20 \mu \mathrm{g})$ from SVTT1 and clone 5 cells with antibodies against p53, AIF and tubulin (as a cytoplasmic marker). (E) Immunofluorescence of cells stained with p53 and AIF antibodies. Colocalization is indicated in white. In $\mathbf{C}$ and $\mathbf{E}$, visualization was performed by confocal microscopy. NIH3T3, NIH; SVTT1, SV; clone 5, cl.5; clone 6, cl.6; clone 7, cl.7. Scale bars in $\mathbf{C}$ and $\mathbf{E}$ indicate $10 \mu \mathrm{m}$. 


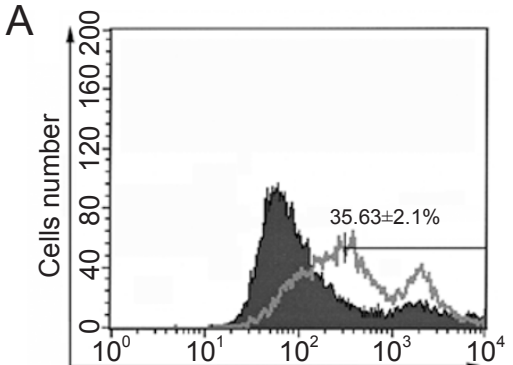

Fluorescence intensity-FITC

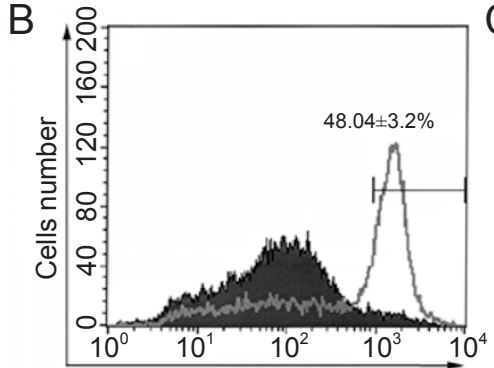

Fluorescence intensity-FITC

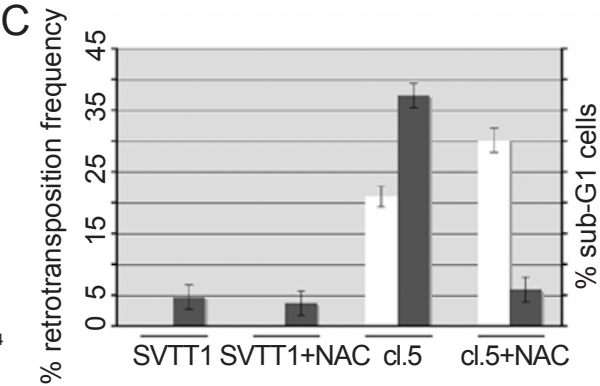

Figure 5 The involvement of mitochondria and lysosomes in VL30 retrotransposition-induced cell death. (A and B) Representative fluorescence profiles overlay of SVTT1 (solid black) and clone 5 (gray line) cells labeled either with JC-1 or acridine orange, respectively, and measured with FACS. Bar denotes threshold for increased green fluorescence. Numerical values represent net percentage of clone 5 cells with $\Delta \psi_{\mathrm{m}}$ loss and LMP as a mean value $(n=3)$ with SD $( \pm)$. (C) Retrotransposition frequency (white column) and cell death rate (black column) mean values $(n=3)$ of untreated cells or those treated with 10 mM NAC for $24 \mathrm{~h}$. SD is indicated with bars.

tantly, in clone 5 cells, while p53 was localized in both the mitochondria and nucleus, AIF was strictly localized to the mitochondria as in parental SVTT1 cells, hence excluding its involvement in cell death (Figure 4E). Mitochondrial localization of $\mathrm{p} 53$ and the absence of AIF release in clone 5 cells were further confirmed by immunoblotting (Figure 4D).

Mitochondrial outer membrane permeabilization (MOMP) constitutes one of the major checkpoints of cell death [32], and is shown in several cases to be caused by p53 translocation [33]. Moreover, MOMP is interrelated to lysosomal rupture [15], which is induced by a low steady-state concentration of $\mathrm{H}_{2} \mathrm{O}_{2}$ [34]. In this context, we first assessed the mitochondrial integrity of clone 5 cells by measuring mitochondrial membrane potential
$\left(\Delta \psi_{\mathrm{m}}\right)$ by JC-1 staining, which assays the potential over the inner mitochondrial membrane. Remarkably, we found a highly statistically significant shift ( $35 \%$ of cells) from red to green fluorescence (Figure 5A) compared to the control cells, demonstrating membrane depolarization due to loss of mitochondrial integrity. Next, the lysosomal integrity of clone 5 cells was measured by acridine orange staining. We found $48 \%$ cells with higher green cytosolic fluorescence compared to control cells, documenting a significant lysosomal rupture of clone 5 cells (Figure 5B). In addition, by measuring lysosomal integrity for clones 6 and 7, we also found increased green fluorescence percentages of $11.2 \%$ and $18.3 \%$, respectively. Interestingly, all three values correlated with the extent of retrotransposition outcomes shown above for induced
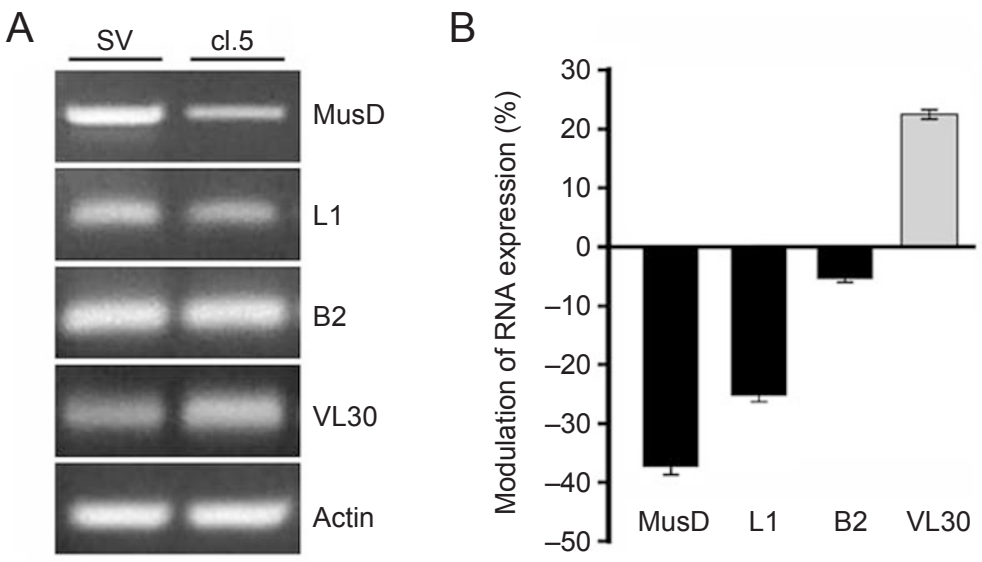

Figure 6 Specificity of VL30 retrotransposition-mediated cell death. (A) RT-PCR of retrotransposon RNA expression performed with 8 ng cDNA. SV and cl.5 denote SVTT1 and clone 5 cells, respectively. (B) Modulated retrotransposon RNA expression shown in $\mathbf{A}$. Columns represent densitometry values of expression normalized to actin and compared to that of SVTT1. Error bars represent standard deviation from three independent experiments. 
sub-G1 populations (Figure 1C), DNA fragmentation (Figure 2B) and p53 induction (Figure 4A and 4B), suggesting a lysosomal damage-dependent cell death mechanism. Finally, given that the mitochondria are the major reactive oxygen species (ROS)-generating organelles and that the best studied inducers of LMP are ROS [15], we asked whether cell death was due to mitochondrialderived ROS. To this end, clone 5 cells were measured both for retrotransposition frequency and cell death rate following treatment with the general antioxidant $\mathrm{N}$-acetylcysteine (NAC). While there was an $85 \%$ decrease in cell death, the retrotransposition frequency was increased by $\sim 33 \%$ (Figure $5 \mathrm{C}$ ). Taken together, these results support the direct involvement of mitochondrial-generated ROS and concomitant lysosomal damage in cell death mediated by VL30 retrotransposition.

\section{VL30 retrotransposition-mediated cell death is VL30 ret- rotransposon specific}

Finally, we asked whether retrotransposition events from other retrotransposon families contributed to the observed cell death. Since an RNA-intermediate is required for the occurrence of a retrotransposition event [12], we indirectly investigated the mobilization of nonLTR L1, B2 and LTR MusD retrotransposons through RNA expression. Interestingly, RT-PCR analysis showed that their RNA expression decreased in clone 5 as compared to SVTT1 cells (Figure 6A). Using densitometry analysis, we found a decrease of $37.9 \%, 24.1 \%$ and $5.85 \%$ for MusD, L1 and B2 expression, respectively. In contrast, the RNA expression of VL30s was increased by $23 \%$ (Figure $6 \mathrm{~B}$ ), suggesting that the induction of cell death was a VL30-specific effect.

\section{Discussion}

Our results provide strong evidence that VL30 retrotransposition specifically induces a caspase-independent and p53-dependent cell death manifested by activation of the mitochondrial death pathway, including lysosomal damage. Evidence supporting our conclusions emerged from three major findings.

First, we found that under VL30 retrotranspositioninduced cell death conditions the RNA expression of VL30s was induced (Figure 6), consistent with our previous finding using the apoptosis-inducer $\mathrm{C}_{2}$-ceramide [3], whereas that of retrotransposons L1, B2 and MusD was reduced. Notably, L1s mediate retrotransposition of other non-LTR retrotransposons such as B2 [35] and LTR retrotransposons MusDs trigger in trans retrotransposition of LTR retrotransposons ETns [36], constituting in sum $\sim 70 \%$ of the total retrotransposon load of the mouse ge- nome [1]. These data and our present findings suggest the specificity of VL30s in mediating cell death, as induced VL30 transcripts may: (a) maintain the retrotransposition events until the late stages of death, and (b) contribute, to some extent, to inhibiting the translation machinery as demonstrated under stress conditions [11].

Second, there was a lack of caspase activation, which was supported by three different assays used (Figure 3 and Supplementary information, Figure S1) and, moreover, an involvement of oxidative stress (Figure 5). Lack of caspase activation may be attributed to LT expression, consistent with previous findings on oxidative stress-induced death of SV40-transformed NIH3T3 cells [28]. In addition, it has been reported that continuous exposure to $\mathrm{H}_{2} \mathrm{O}_{2}$ at low concentrations inactivates procaspase- 9 and inhibits caspase-dependent apoptosis [37]. This further supports our finding of caspase-independent cell death, since the induction of MOMP (Figure 5A) implicates an increase of intracellular $\mathrm{H}_{2} \mathrm{O}_{2}$ in our assay conditions. Moreover, the absence of the release of AIF (Figure $4 \mathrm{E}$ ) and the pronounced lysosomal leakage (Figure 5B) provided additional support for the lack of caspase activation $[32,38]$, characterizing the VL30-mediated cell death in SV40-transformed cells.

Third, we found two interrelated critical events in the induction of cell death, namely: induction of VL30 retrotransposition and p53-dependent responses. Regarding the former, it was shown that cell death correlated with the extent of retrotransposition events (Figure 1B and $1 \mathrm{C}$ ). A natural retrotransposition frequency for an LTR retrotransposon, such as VL30, was calculated as $10^{-6}$ events per cell per generation [39]. Thus a value of $22.6 \%$ found for clone $5-226000$-fold higher than the natural retrotransposition frequency and the corresponding 39\% death rate - mirror the extent of the endonuclease cuts required for new VL30 copy-integration which would lead to genome damage. Accordingly, these data establish VL30 retrotransposition as a primary stimulus of genome damage. Two lines of evidence support the dependency of retrotransposition-mediated cell death on p53 action. First, we found trans-activation of p53-target genes: Bcl-2 downregulation, PUMA $\alpha$ induction and Bax induction/oligomerization (Figure 4B) correlated with p53 induction (Figure 4A) and second, translocation of p53 to mitochondria (Figure 4D and 4E), both targeting mitochondria for cell death induction [33]. As evident from Figure 4D, less of the induced p53 was found in mitochondria than in the cytosol. This is consistent with a previous observation that only a fraction of induced p53 protein specifically localizes to the mitochondria in p53-mediated death, contributing to MOMP in response to DNA damage [31]. In keeping with this, we propose 
that the observed loss of mitochondrial integrity (Figure $5 \mathrm{~A}$ ) allowed the release of mitochondrial ROS contributing to lysosomal damage (Figure 5B). The latter is also supported by the observed Hsp70 downregulation (Figure 4B), a known safeguard of lysosomal integrity [40]. On the other hand, the abolishment of cell death following NAC treatment (Figure 5C) provides additional evidence for the direct involvement of mitochondrial-generated ROS, acting as an execution factor in retrotranspositionmediated cell death. Collectively, these major findings uncover: (i) a functional role of VL30 retrotransposition as a signal in mediating cell death through the activation of the mitochondrial death pathway, and (ii) a crosstalk between the nucleus, as a signaling organelle through retrotransposons, and the mitochondrial-lysosomal axis.

VL30s, as non-coding RNA, play an important role in mouse cell physiology by interacting with the major tumor-suppressor protein PSF $[9,10]$. Our tagged construct of VL30 [22] is truncated for $\sim 2.5 \mathrm{~Kb}$ internal sequences (nucleotides 1 480-4 036) encompassing the critical region (nucleotides 1 523-1 841) that is responsible for binding PSF [9], and is not expected to interact with PSF. Thus, it is reasonable to believe that the primary effects on cell death are consequences of genomic integrations resulting from VL30 retrotransposition events. Additionally, given that increased VL30 transcripts were detected (Figure 6) we do not exclude the possibility that both cis effects of retrotransposition and trans effects of transcription could be responsible for initiating the reactions leading to cell death.

Based on our findings, we propose a two-step mechanism in VL30-mediated cell death. First, LT stimulates VL30 retrotransposition [22], and continuously accumulated VL30 integration events result in genome damage leading to the induction of $\mathrm{p} 53$, which in turn regulates anti-apoptotic and pro-apoptotic target genes (Figure 4A and $4 \mathrm{~B}$ ) and translocates to mitochondria (Figure 4D and $4 \mathrm{E}$ ). Second, mitochondrial disruption (Figure 5A) by p53 action allows the release of ROS, which is responsible for lysosomal damage (Figure 5B) and VL30 retrotransposition enhancement by $\mathrm{H}_{2} \mathrm{O}_{2}$ [23], forming two positive feedback loops. Ultimately, the highly active ROS cause further DNA damage, which together with the activation of lysosomal proteases leads to cell death. Additionally, we believe that future analyses of chromosomal loci or genes affected by the accumulated integration events might reveal inactivated survival genes, which could also contribute to the observed cell death.

An important feature of our study was the expression of LT, a constant stimulus for VL30 retrotransposition, and cell death of long-term living cells (clones) signifies the reliability of our findings. It has been suggested that the classification of several death types using exclusive definitions may be somewhat artificial due to the overlap and shared signaling pathways between different death programs [17]. This study supports the aforementioned suggestion as the death type studied encompassed hallmarks of: (a) apoptosis (nuclear condensation and DNA fragmentation), (b) necrosis-like death (cytoplasm vacuolization), and (c) mitotic catastrophe (cell multinucleation) (Figure 2B and 2C). We believe that DNA fragmentation is a primary effect of accumulated VL30 integration as exemplified by L1 retrotransposition, estimated to induce 10 - to 100 -fold more DSBs than the predicted numbers of successful integrations [41]. Given that DNA damage constitutes a basic inducer of mitotic catastrophe [42], such VL30 retrotransposition-induced DSBs might explain multinucleation, a feature of mitotic catastrophe (Figure 2C). The observed induction of MOMP and LMP as well as the decrease of cell death rate following the use of the antioxidant NAC signified the induction of ROS in retrotransposition-positive cells (Figure 5C). Based on this, necrosis-like death may be explained by $\mathrm{H}_{2} \mathrm{O}_{2}$ release following mitochondrial damage (Figure 5A) and/or LMP (Figure 5B), as suggested by others [43]. Accordingly, it is reasonable to suggest that VL30 retrotransposition mediates cell death with parallel or overlapping characteristics of a complex but integral death phenomenon.

We earlier showed that both metal-generated oxidative stress [23] and the oncogenic SV40 virus [22], constituting threats against a cell's normal life, induced VL30 retrotransposition, which was associated with cytotoxic effects in normal NIH3T3 cells. While control of retrotransposition is essential for cell homeostasis, the present study shows that deregulation of retrotransposition leads to a homeostatic imbalance, and eventually to cell death. In her Nobel Prize acceptance lecture in 1983, Barbara McClintock's last sentence was: 'We know nothing, however, about how the cell senses danger and instigates responses to it'. Here we demonstrate that retrotransposons, previously thought of as inert sequences, are significant controlling elements in signaling pathways that determine the fate of cells.

\section{Materials and Methods}

\section{Cell culture}

SVTT1 (SVLTR1), SV40-transformed NIH3T3 mouse embryo fibroblasts [44] and NIH3T3 cells were grown in D-MEM medium supplemented with $10 \%$ FCS, $100 \mathrm{U} / \mathrm{ml}$ penicillin and $100 \mu \mathrm{g} / \mathrm{ml}$ streptomycin.

Plasmid DNA transfection, cell cloning and clonogenic assay NIH3T3 or SVTT1 cells were transfected (Qiagen) with 2.5 
$\mu \mathrm{g}$ of pEGFP-N1 (Clontech) or pNVL-3*/EGFP-INT [22] DNA. Single clones were isolated following selection with $400 \mu \mathrm{g} / \mathrm{ml}$ G418 or $80 \mu \mathrm{g} / \mathrm{ml}$ hygromycin B, respectively, for 18 days. Measurement of clonogenic frequency was performed in 10-cm dishes following selection of clones fixed and stained with Giemsa. Student's $t$-test was used for statistical analysis.

Measurement of retrotransposition frequency, mitochondrial membrane potential and lysosomal damage and cell cycle analysis

Measurement of retrotransposition frequency [22], lysosomal integrity by acridine orange staining [34] and mitochondrial membrane potential through JC-1 or cell cycle analysis using BD MitoScreen or CycleTEST Kits (Becton Dickinson), respectively, were performed with FACS. Data were analyzed with CellQuest v.3.1 software.

\section{PCR and RT-PCR analysis, DNA fragmentation and fluores-} cence microscopy

Protocols described were used for PCR [22], DNA fragmentation analysis [45] and fluorescence microscopy [22]. RT-PCR was performed [23] with 8 ng cDNA using MusD- [46] or VL30-, L1and B2- [47] specific primers.

\section{Immunoblotting}

Immunoblotting of sub-cellular fractionated [48] or total proteins was performed using monoclonal antibodies to p53, Bcl-2, Bax and AIF (Santa Cruz); cleaved caspase-3 (Cell Signaling); Hsp70 (Stressgen); $\alpha$-tubulin and $\beta$-actin (Sigma); and using polyclonal antibodies to PUMA $\alpha$ (Santa Cruz) and Lamin B [49]. Protein detection was performed by chemiluminescence (Pierce), while induction of expression was measured by densitometry (Kodak) normalized to $\beta$-actin signals.

\section{Indirect immunofluorescence analysis}

Cells fixed in $4 \%$ formaldehyde were reacted with anti-p53 (Santa Cruz) and anti-LT (Calbiochem) antibodies followed by donkey anti-goat IgG/TRITC (Alexa) and anti-mouse IgG/Cy-5 (Dianova) secondary antibodies. For Lamin B immunofluorescence, cells fixed with $1 \%$ formaldehyde were reacted with a Lamin B polyclonal [49] and secondary donkey anti-rabbit IgG/ Cy-5 (Dianova) antibodies. Treated cells incubated with RNase A $(1 \mathrm{mg} / \mathrm{ml})$ for $30 \mathrm{~min}$ were stained with PI $(10 \mu \mathrm{g} / \mathrm{ml})$ for 8 min. For p53 and AIF immunofluorescence, cells were fixed with $100 \%$ methanol, reacted with primary p53, AIF (Santa Cruz), secondary anti-goat/TRITC (Alexa) and anti-mouse/FITC (Sigma) antibodies. Specimens were observed with a Leica SP confocal microscope. ImageJ $1.34 \mathrm{~s}$ software and colocalization finder plugin (http://rsb.info.nih.gov/ij/plugins/) were used for colocalization and scatter plot analysis.

\section{Measurement of caspase-3-like activity}

Measurement of caspase-3-like activity was performed with $150 \mu \mathrm{g}$ of total protein extract using Ac-DEVD-AMC as substrate in a fluorospectrophotometric assay [50].

\section{Acknowledgments}

We thank Professors Sten Orrenius (Division of Toxicol- ogy, Institute of Environmental Medicine, Karolinska Institutet, Stockholm, Sweden) and Ulf Brunk (Pharmacology, Faculty of Health Sciences, University of Linköping, University Hospital, Linköping, Sweden) for their comments and for reviewing the manuscript.

\section{References}

1 Waterston RH, Lindblad-Toh K, Birney E, et al. Initial sequencing and comparative analysis of the mouse genome. Nature 2002; 420:520-562.

2 French NS, Norton JD. Structure and functional properties of mouse VL30 retrotransposons. Biochim Biophys Acta 1997; 1352:33-47.

3 Tzavaras T, Eftaxia S, Tavoulari S, Hatzi P, Angelidis C. Factors influencing the expression of endogenous reverse transcriptases and viral-like 30 elements in mouse NIH3T3 cells. Int J Oncol 2003; 23:1237-1243.

4 Tzavaras T, Kalogera C, Eftaxia S, Saragosti S, Pagoulatos GN. Clone-specific high-frequency retrotransposition of a recombinant virus containing a VL30 promoter in SV40-transformed NIH3T3 cells. Biochim Biophys Acta 1998; 1442:186198.

5 Adams SE, Rathjen PD, Stanway CA, et al. Complete nucleotide sequence of a mouse VL30 retro-element. Mol Cell Biol 1988; 8:2989-2998.

6 Song X, Wang B, Bromberg M, Hu Z, Konigsberg W, Garen A. Retroviral-mediated transmission of a mouse VL30 RNA to human melanoma cells promotes metastasis in an immunodeficient mouse model. Proc Natl Acad Sci USA 2002; 99:62696273.

7 Song X, Sun Y, Garen A. Roles of PSF protein and VL30 RNA in reversible gene regulation. Proc Natl Acad Sci USA 2005; 102:12189-12193.

8 Garen A, Song X. Regulatory roles of tumor-suppressor proteins and noncoding RNA in cancer and normal cell functions. Int J Cancer 2008; 122: 1687-1689.

9 Song X, Sui A, Garen A. Binding of mouse VL30 retrotransposon RNA to PSF protein induces genes repressed by PSF: effects on steroidogenesis and oncogenesis. Proc Natl Acad Sci USA 2004; 101: 621-626.

10 Wang G, Cui Y, Zhang G, Garen A, Song X. Regulation of proto-oncogene transcription, cell proliferation, and tumorigenesis in mice by PSF protein and a VL30 noncoding RNA. Proc Natl Acad Sci USA 2009; 106:16794-16798.

11 Costain WJ, Rasquinha I, Graber T, et al. Cerebral ischemia induces neuronal expression of novel VL30 mouse retrotransposons bound to polyribosomes. Brain Res 2006; 1094:24-37.

12 Boeke JD, Garfinkel DJ, Styles CA, Fink GR. Ty elements transpose through an RNA intermediate. Cell 1985; 40:491500 .

13 Ostertag EM, Kazazian HH Jr. Biology of mammalian L1 retrotransposons. Annu Rev Genet 2001; 35:501-538.

14 Shapiro JA. Genome system architecture and natural genetic engineering in evolution. Ann N Y Acad Sci 1999; 870:23-35.

15 Kroemer G, Galluzzi L, Brenner C. Mitochondrial membrane permeabilization in cell death. Physiol Rev 2007; 87:99-163.

16 Orrenius S, Gogvadze V, Zhivotovsky B. Mitochondrial oxidative stress: implications for cell death. Annu Rev Pharmacol 
Toxicol 2007; 47:143-183.

17 Broker LE, Kruyt FA, Giaccone G. Cell death independent of caspases: a review. Clin Cancer Res 2005; 11:3155-3162.

18 Servomaa K, Rytomaa T. Suicidal death of rat chloroleukaemia cells by activation of the long interspersed repetitive DNA element (L1Rn). Cell Tissue Kinet 1988; 21: 33-43.

19 Servomaa K, Rytomaa T. UV light and ionizing radiations cause programmed death of rat chloroleukaemia cells by inducing retropositions of a mobile DNA element (L1Rn). Int $J$ Radiat Biol 1990; 57:331-343.

20 Haoudi A, Semmes OJ, Mason JM, Cannon RE. Retrotransposition-competent human LINE-1 induces apoptosis in cancer cells with intact p53. J Biomed Biotechnol 2004; 2004:185194.

21 Belgnaoui SM, Gosden RG, Semmes OJ, Haoudi A. Human LINE-1 retrotransposon induces DNA damage and apoptosis in cancer cells. Cancer Cell Int 2006; 6:13.

22 Noutsopoulos D, Vartholomatos G, Kolaitis N, Tzavaras T. SV40 large $\mathrm{T}$ antigen up-regulates the retrotransposition frequency of viral-like 30 elements. J Mol Biol 2006; 361:450461.

23 Noutsopoulos D, Markopoulos G, Koliou M, et al. Vanadium induces VL30 retrotransposition at an unually high level: a possible carcinogenesis mechanism. J Mol Biol 2007; 374:8090.

24 Nicholson DW, Ali A, Thornberry NA, et al. Identification and inhibition of the ICE/CED-3 protease necessary for mammalian apoptosis. Nature 1995; 376:37-43.

25 Slee EA, Adrain C, Martin SJ. Executioner caspase-3, -6, and -7 perform distinct, non-redundant roles during the demolition phase of apoptosis. J Biol Chem 2001; 276:7320-7326.

26 Ruchaud S, Korfali N, Villa P, et al. Caspase-6 gene disruption reveals a requirement for lamin A cleavage in apoptotic chromatin condensation. EMBO J 2002; 21:1967-1977.

27 Oren M. Decision making by p53: life, death and cancer. Cell Death Differ 2003; 10:431-442.

28 Gonin S, Diaz-Latoud C, Richard MJ, et al. p53/T-antigen complex disruption in T-antigen transformed NIH3T3 fibroblasts exposed to oxidative stress: correlation with the appearance of a Fas/APO-1/CD95 dependent, caspase independent, necrotic pathway. Oncogene 1999; 18:8011-8023.

29 Zinkel S, Gross A, Yang E. BCL2 family in DNA damage and cell cycle control. Cell Death Differ 2006; 13:1351-1359.

30 Quenneville LA, Trotter MJ, Maeda T, Tron VA. p53-dependent regulation of heat shock protein 72. Br J Dermatol 2002; 146: 786-791.

31 Erster S, Mihara M, Kim RH, Petrenko O, Moll UM. In vivo mitochondrial p53 translocation triggers a rapid first wave of cell death in response to DNA damage that can precede p53 target gene activation. Mol Cell Biol 2004; 24:6728-6741.

32 Boya P, Kroemer G. Lysosomal membrane permeabilization in cell death. Oncogene 2008; 27:6434-6451.

33 Moll UM, Marchenko N, Zhang XK. p53 and Nur77/TR3 transcription factors that directly target mitochondria for cell death induction. Oncogene 2006; 25:4725-4743.

34 Antunes F, Cadenas E, Brunk UT. Apoptosis induced by exposure to a low steady-state concentration of $\mathrm{H}_{2} \mathrm{O}_{2}$ is a consequence of lysosomal rupture. Biochem J 2001; 356:549-555.
35 Dewannieux M, Heidmann T. L1-mediated retrotransposition of murine B1 and B2 SINEs recapitulated in cultured cells. $J$ Mol Biol 2005; 349:241-247.

36 Ribet D, Dewannieux M, Heidmann T. An active murine transposon family pair: retrotransposition of 'master' MusD copies and ETn trans-mobilization. Genome Res 2004; 14: 2261-2267.

37 Barbouti A, Amorgianiotis C, Kolettas E, Kanavaros P, Galaris D. Hydrogen peroxide inhibits caspase-dependent apoptosis by inactivating procaspase-9 in an iron-dependent manner. Free Radic Biol Med 2007; 43:1377-1387.

38 Arnoult D, Gaume B, Karbowski M, Sharpe JC, Cecconi F, Youle RJ. Mitochondrial release of AIF and EndoG requires caspase activation downstream of Bax/Bak-mediated permeabilization. EMBO J 2003; 22:4385-4399.

39 Heidmann T, Heidmann O, Nicolas JF. An indicator gene to demonstrate intracellular transposition of defective retroviruses. Proc Natl Acad Sci USA 1988; 85:2219-2223.

40 Gyrd-Hansen M, Nylandsted J, Jaattela M. Heat shock protein 70 promotes cancer cell viability by safeguarding lysosomal integrity. Cell Cycle 2004; 3:1484-1485.

41 Gasior SL, Wakeman TP, Xu B, Deininger PL. The human LINE-1 retrotransposon creates DNA double-strand breaks. $J$ Mol Biol 2006; 357:1383-1393.

42 Vakifahmetoglu H, Olsson M, Zhivotovsky B. Death through a tragedy: mitotic catastrophe. Cell Death Differ 2008; 15:1153-1162.

43 Kurz T, Terman A, Brunk UT. Autophagy, ageing and apoptosis: the role of oxidative stress and lysosomal iron. Arch Biochem Biophys 2007; 462:220-230.

44 Kriegler M, Botchan M. Enhanced transformation by a simian virus 40 recombinant virus containing a Harvey murine sarcoma virus long terminal repeat. Mol Cell Biol 1983; 3:325339.

45 Sugiki H, Hozumi Y, Maeshima H, Katagata Y, Mitsuhashi $\mathrm{Y}$, Kondo $\mathrm{S} . \mathrm{C}_{2}$-ceramide induces apoptosis in a human squamous cell carcinoma cell line. Br J Dermatol 2000; 143:1154-1163.

46 Baust C, Gagnier L, Baillie GJ, Harris MJ, Juriloff DM, Mager DL. Structure and expression of mobile ETnII retroelements and their coding-competent MusD relatives in the mouse. J Virol 2003; 77:11448-11458.

47 Puschendorf M, Stein P, Oakeley EJ, Schultz RM, Peters AH, Svoboda P. Abundant transcripts from retrotransposons are unstable in fully grown mouse oocytes. Biochem Biophys Res Commun 2006; 347:36-43.

48 Eom YW, Kim MA, Park SS, et al. Two distinct modes of cell death induced by doxorubicin: apoptosis and cell death through mitotic catastrophe accompanied by senescence-like phenotype. Oncogene 2005; 24:4765-4777.

49 Maison C, Horstmann H, Georgatos SD. Regulated docking of nuclear membrane vesicles to vimentin filaments during mitosis. J Cell Biol 1993; 123:1491-1505.

50 Barbouti A, Doulias PT, Nousis L, Tenopoulou M, Galaris D. DNA damage and apoptosis in hydrogen peroxide-exposed Jurkat cells: bolus addition versus continuous generation of $\mathrm{H}_{2} \mathrm{O}_{2}$. Free Radic Biol Med 2002; 33:691-702.

(Supplementary information is linked to the online version of the paper on the Cell Research website.) 